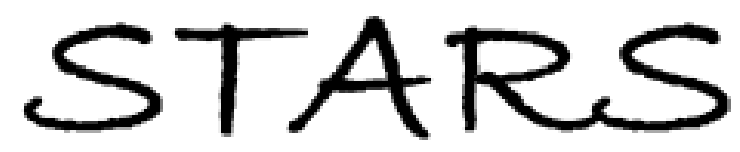

University of Central Florida

STARS

$1-1-2012$

\title{
Enhanced algorithm based on persistent scatterer interferometry for the estimation of high-rate land subsidence
}

\author{
Zahra Sadeghi \\ Mohammad Javad Valadan Zoej \\ Maryam Dehghami \\ Ni-Bin Chang \\ University of Central Florida
}

Find similar works at: https://stars.library.ucf.edu/facultybib2010

University of Central Florida Libraries http://library.ucf.edu

This Article is brought to you for free and open access by the Faculty Bibliography at STARS. It has been accepted for inclusion in Faculty Bibliography 2010 s by an authorized administrator of STARS. For more information, please contactSTARS@ucf.edu.

\section{Recommended Citation}

Sadeghi, Zahra; Zoej, Mohammad Javad Valadan; Dehghami, Maryam; and Chang, Ni-Bin, "Enhanced algorithm based on persistent scatterer interferometry for the estimation of high-rate land subsidence" (2012). Faculty Bibliography 2010s. 3223.

https://stars.library.ucf.edu/facultybib2010/3223

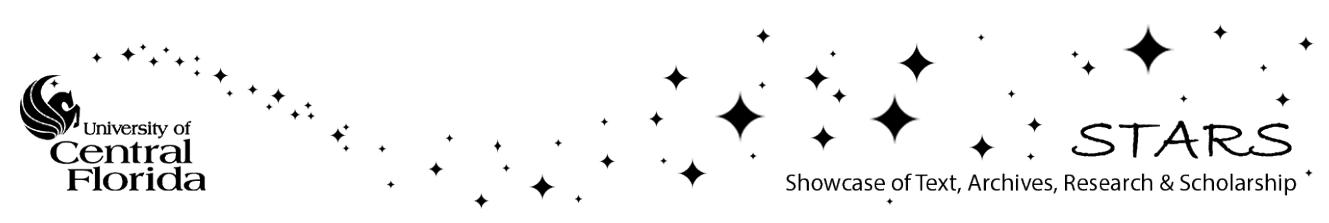


Applied Remote Sensing

\section{Enhanced algorithm based on persistent scatterer interferometry for the estimation of high-rate land subsidence}

Zahra Sadeghi

Mohammad Javad Valadan Zoej

Maryam Dehghani

Ni-Bin Chang 


\title{
Enhanced algorithm based on persistent scatterer interferometry for the estimation of high-rate land subsidence
}

\author{
Zahra Sadeghi, ${ }^{a}$ Mohammad Javad Valadan Zoej, ${ }^{\text {a }}$ Maryam Dehghani, ${ }^{\text {b }}$ \\ and Ni-Bin Chang \\ ${ }^{a}$ K.N. Toosi University of Technology (KNTU), Faculty of Geomatics and Geodesy, \\ Tehran 11369, Iran \\ ${ }^{\mathrm{b}}$ Shiraz University, Department of Civil and Environmental Engineering, School of Engineering, \\ Shiraz 71364, Iran \\ ${ }^{\mathrm{c}}$ University of Central Florida, Civil, Environmental, and Construction Engineering Department, \\ Orlando, Florida 32816 \\ nchang@mail.ucf.edu
}

\begin{abstract}
Persistent scatterer interferometry (PSI) techniques using amplitude analysis and considering a temporal deformation model for PS pixel selection are unable to identify PS pixels in rural areas lacking human-made structures. In contrast, high rates of land subsidence lead to significant phase-unwrapping errors in a recently developed PSI algorithm (StaMPS) that applies phase stability and amplitude analysis to select the PS pixels in rural areas. The objective of this paper is to present an enhanced algorithm based on PSI to estimate the deformation rate in rural areas undergoing high and nearly constant rates of deformation. The proposed approach integrates the strengths of all of the existing PSI algorithms in PS pixel selection and phase unwrapping. PS pixels are first selected based on the amplitude information and phase-stability estimation as performed in StaMPS. The phase-unwrapping step, including the deformation rate and phase-ambiguity estimation, is then performed using least-squares ambiguity decorrelation adjustment (LAMBDA). The atmospheric phase screen (APS) and nonlinear deformation contribution to the phase are estimated by applying a high-pass temporal filter to the residuals derived from the LAMBDA method. The final deformation rate and the ambiguity parameter are re-estimated after subtracting the APS and the nonlinear deformation from that of the initial phase. The proposed method is applied to 22 ENVISAT ASAR images of southwestern Tehran basin captured between 2003 and 2008. A quantitative comparison with the results obtained with leveling and GPS measurements demonstrates the significant improvement of the PSI technique. (C) 2012 Society of Photo-Optical Instrumentation Engineers (SPIE). [DOI: 10.1117/1.JRS.6.063573]
\end{abstract}

Keywords: land subsidence; interferometric synthetic aperture radar; persistent scatterer; high deformation rate; least-squares ambiguity decorrelation adjustment..

Paper 11274 received Dec. 28, 2011; revised manuscript received Jul. 12, 2012; accepted for publication Aug. 15, 2012; published online Sep. 12, 2012.

\section{Introduction}

Monitoring the deformation rate of ground subsidence is critical for hazard management. Interferometric synthetic aperture radar (InSAR) provides precise measurements of surface deformation caused by land subsidence. However, temporal and geometrical decorrelation often prevents conventional interferometry from being an operational tool for surface deformation monitoring. Persistent (or permanent) scatterer interferometry (PSI) is a recently developed technique used to address temporal and spatial decorrelations by identifying pixels with persistent scattering behavior in time. Several PSI algorithms have been introduced to identify PS pixels and estimate deformation rates. Although each algorithm has a wide range of advantages, each faces various limitations. One of these algorithms, developed by Delft University of 
Technology, is the Delft implementation for persistent scatterer interferometry (DePSI), in which PS pixels with small amplitude dispersion are selected. ${ }^{1,2}$ DePSI can be successfully performed in areas containing large numbers of high-amplitude scatterers provided by human-made structures. ${ }^{1,2}$ However, this algorithm fails to identify PS pixels in rural areas lacking bright scatterers because of the use of amplitude analysis only in the PS identification step. This algorithm is further limited because only scatterers with a deformation behavior close to a predefined functional model can be identified as PS pixels, and such a predefined model might not be available in most cases.

Hooper $^{3}$ introduced a new PSI method, the Stanford method for PS (StaMPS), which uses the spatial correlation between pixels to identify PS pixels and estimate the deformation rate. As no $a$ priori temporal model of the deformation is required for the identification of PS pixels, this approach is applicable in areas undergoing unsteady deformation. Moreover, PS pixels in areas lacking human-made structures can be detected using amplitude analysis followed by phasestability estimation. However, accurate phase unwrapping in StaMPS is possible only when the absolute phase difference between nearby PS pixels in time or space is less than $\pi .^{3,4}$ This limitation leads to the violation of the Nyquist sampling assumption and consequently the underestimation of the deformation rate in areas with a high deformation rate or poor temporal sampling of data. ${ }^{5,6}$

Dehghani et al. ${ }^{6,7}$ presented a hybrid method of conventional interferometry and PSI to increase the performance of StaMPS for monitoring land subsidence in rural areas with high deformation rates. To reduce the deformation rate and fulfill the Nyquist sampling criterion, the linear component of deformation, which is much more significant than the seasonal component, is subtracted from the wrapped phase of each interferogram. The linear component is extracted by stacking a number of coherent interferograms that can be spatially unwrapped. ${ }^{8}$ After unwrapping the residual phase, based on the Nyquist assumption, the linear term is added back to the unwrapped phase. However, the drawback of this method is the limited availability of radar data with good temporal sampling frequencies and coherent interferograms for linear term estimation.

Wegmuller employed TerraSAR-X data with higher spatial resolution and shorter repeat intervals to improve the applicability of PSI for high-deformation-rate measurements. ${ }^{9}$ The spatial phase unwrapping is significantly improved by the dense candidate PS network provided by the high spatial resolution. Moreover, the complexity of the phase unwrapping is reduced by small time intervals, which decrease the quantity of deformation occurring between two data acquisitions. This method is applicable whenever TerraSAR-X data are available, which is probably not always the case.

The primary purpose of this paper is to present an enhanced PSI (EPSI) algorithm that combines the merits of different algorithms to enable the application of PSI for a difficult test case, i.e., a rural area with a high deformation rate and poor temporal sampling of the data. The proposed method applies the amplitude and phase analysis used in StaMPS for PS identification. However, it also uses the LAMBDA method applied in DePSI for temporal phase unwrapping. This can enable us to accurately estimate high, nearly constant deformation rates in rural areas lacking human-made features. The proposed method was applied to an area subject to land subsidence induced by the overexploitation of groundwater.

The study area is located in the southwestern Tehran basin, primarily covered by agricultural fields (Fig. 1). Because the area is highly subject to temporal and spatial decorrelation due to the poor sampling of the radar dataset, conventional small baseline subset (SBAS) interferometry fails to measure the deformation. Moreover, neither the DePSI nor StaMPS algorithms can be applied to the area due to the lack of human-made structures and the high deformation rate, respectively. ${ }^{6,7}$ Thus, using the EPSI method, we could successfully estimate the high deformation rates in the southwestern Tehran basin.

\section{Enhanced Algorithm of Persistent Scatterer Interferometry}

The EPSI algorithm, presented in Fig. 2, contains two main parts, each discussed in detail in Secs. 2.1 and 2.2-2.4. The first part, including the PS identification and spatially uncorrelated 


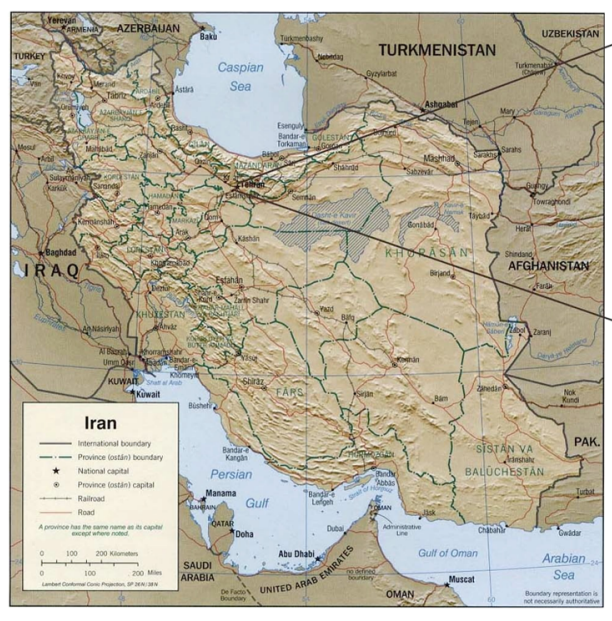

(a)
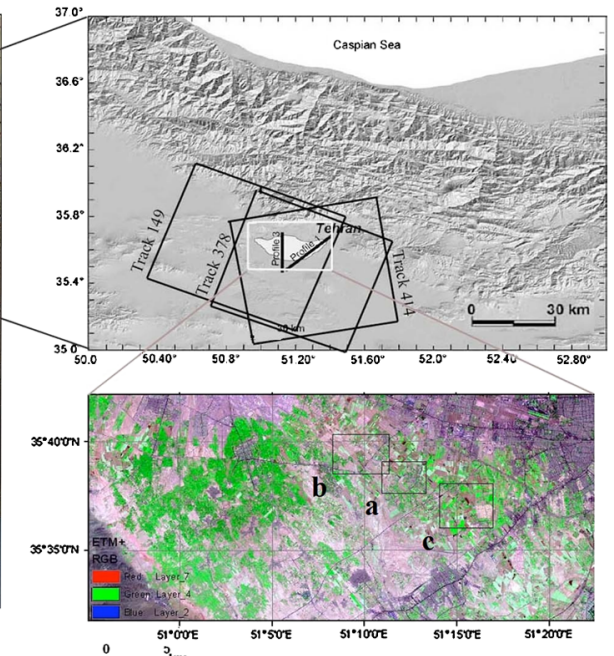

(b)

Fig. 1 (a), Location of the Tehran basin in Iran. (b), Upper right: location of the available ENVISAT ASAR data tracks (black squares) covering the Tehran basin under study (white rectangle). Lower right: LANDSAT $7 \mathrm{ETM}^{+}$color composite image (R:7, G:4, B:2) of the Tehran basin, primarily covered by the agricultural fields. Rectangles $a, b$, and $c$ show the locations of the different study areas.

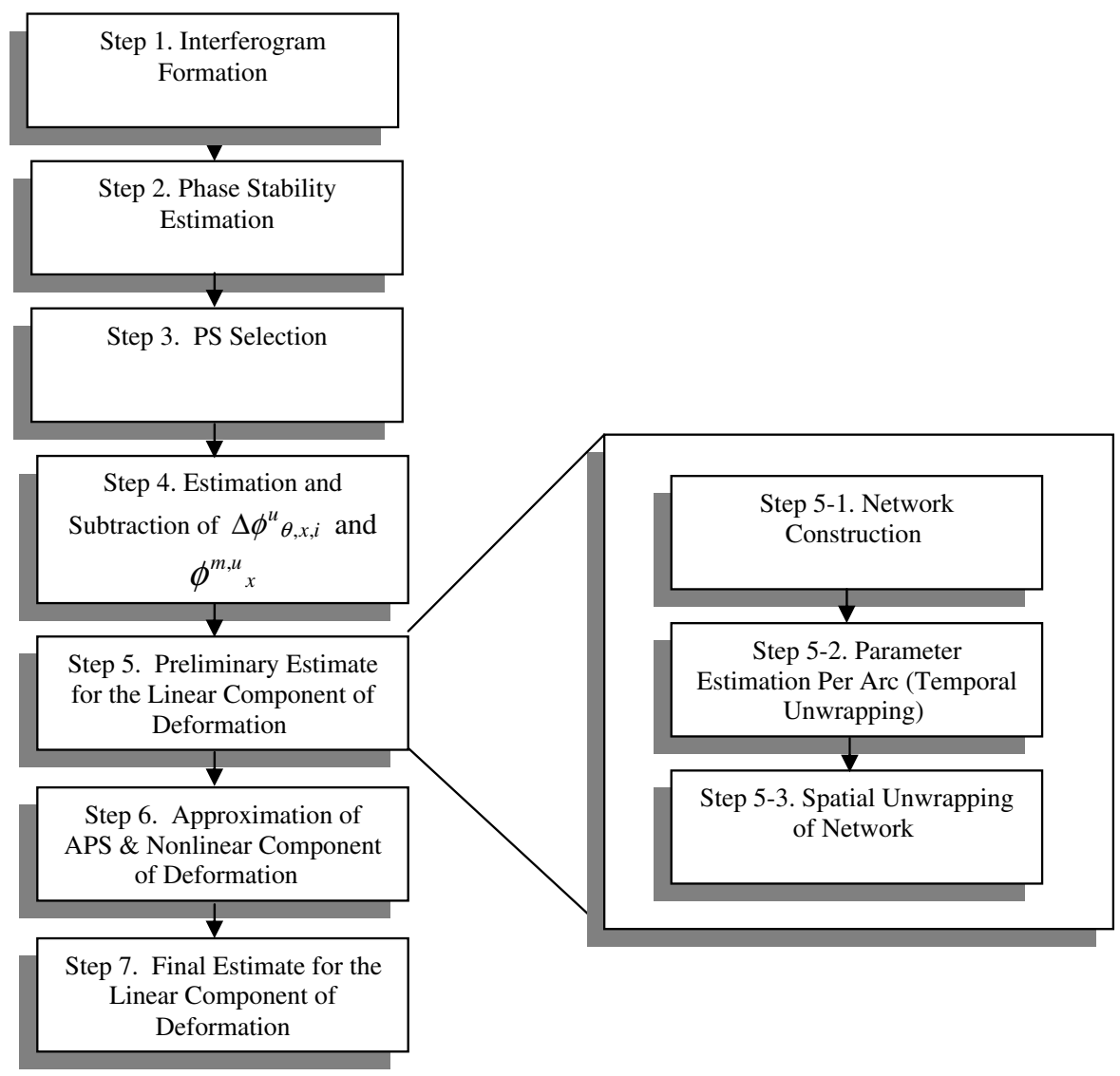

Fig. 2 Flow chart of the enhanced algorithm proposed in this study. 
phase component removal, is performed according to the standard StaMPS method (steps 1-4); the second part includes the phase-unwrapping process and subtraction of APS and non-linear deformation (steps 5-7).

\subsection{PS Selection and Spatially Uncorrelated Phase Removal}

The interferograms are first generated using a single master image maximizing the sum correlation of all interferograms. The interferometric wrapped phase of pixel $x$ of flattened differential interferogram $i$ can be written as a combination of the different contributions as

$$
\psi_{x, i}=W\left\{\phi_{D, x, i}+\phi_{\mathrm{atmo}, x, i}+\Delta \phi_{\mathrm{orbit}, x, i}+\Delta \phi_{\theta, x, i}+\phi_{N, x, i}\right\},
$$

where $\phi_{D, x, i}$ is the phase due to the ground deformation, $\phi_{\mathrm{atmo}, x, i}$ is the atmospheric phase resulting from the different atmospheric delay at the two acquisition times, $\Delta \phi_{\text {orbit }, x, i}$ is the residual phase due to orbital errors, $\Delta \phi_{\theta, x, i}$ is the residual phase due to the look angle error caused by the residual topographic and subpixel position errors, $\phi_{N, x, i}$ is a noise term due primarily to decorrelation, and $w\{\cdot\}$ is the wrapping operator.

The candidate PS pixels were initially selected based on the amplitude dispersion index, which rejects those least likely to be PS pixels. ${ }^{10}$ The phase stability for each candidate was then analyzed in an iterative process. In every iteration, a measure of the phase noise level, which is similar to the measure of coherence in time, i.e., temporal coherence, rather than in space, can be calculated by the estimation and subtraction of the spatially correlated parts of the interferometric phase. The final PS pixels were identified based on the probability of being PS, estimated from the amplitude dispersion and temporal coherence. After the successful selection of PS pixels, the spatially uncorrelated part of the look angle error, $\Delta \phi_{\theta, x, i}^{u}$, and the contribution of the master image to the spatially uncorrelated part of the signal, $\phi_{x}^{m, u}$, were estimated and removed from the original phase before the phase unwrapping. ${ }^{4}$

As mentioned, StaMPS followed the Nyquist sampling assumption for temporal phase unwrapping. ${ }^{4}$ However, this assumption cannot be met when the deformation rate is too high, such that the phase difference between the nearby PS increases by more than $\pi$ in magnitude. ${ }^{6,7}$ In this case, the deformation rate is underestimated. The algorithm presented in this paper tries to use the phase unwrapping method introduced by DePSI, as discussed in Sec. 2.2, which directly estimates the phase ambiguity and the deformation rate from the wrapped phase without assuming the Nyquist sampling criterion.

\subsection{Preliminary Estimate for the Linear Component of Deformation}

The linear component of deformation is much more significant than the seasonal nonlinear one in most areas undergoing land subsidence, such as the southwestern Tehran basin. ${ }^{6,11-13}$ Therefore, we used a linear deformation model in the phase-unwrapping step. In this step, the PS pixels are connected to form a network and make the double-difference measurements as the differenced phase in space and time for each arc connecting two PS pixels. Insofar as it is important to have a redundant network to enable the detection of the unwrapping error,,${ }^{14,15}$ Delaunay triangulation can be used to construct the network. For arc $i j$ in the constructed network, the double-difference wrapped phase observation, $\psi_{i j}^{k}$, between the $i$ th and $j$ th PS in the $k$ th interferogram can be summarized as

$$
\psi_{i j}^{k}=-2 \pi a_{i j}^{k}-\frac{4 \pi}{\lambda} V_{i j} T^{k}+\varphi_{\mathrm{atmo}, i j}^{k}+\varphi_{\mathrm{orbit}, i j}^{k}+\varphi_{\mathrm{nonlinear}, i j}^{k}+\varphi_{N, i j}^{k},
$$

where $a_{i j}^{k}$ is the integer ambiguity, $V_{i j}$ is the relative constant velocity, $\lambda$ is the wavelength, $\varphi_{\mathrm{atmo}, i j}^{k}$ is the atmospheric phase, $\varphi_{\mathrm{orbit}, i j}^{k}$ is the phase of the orbital errors, $\varphi_{\text {nonlinear }, i j}^{k}$ is the phase component due to the nonlinear deformation, $\varphi_{N, i j}^{k}$ is the noise phase, all between the $i$ th and $j$ th PS, $T^{k}$ is the temporal baseline of the $k$ th interferogram, and $-\frac{4 \pi}{\lambda} V_{i j} T^{k}$

is the contribution of the deformation to the phase. All terms for both the left and right sides of Eq. (2) are in radians, while $T^{k}$ is in years. 
The APS, which is the contribution due to the atmospheric phase delay, and the orbital errors are strongly correlated in spatial space. Consequently, the double-difference phase measurements between the nearby PS pixels do not contain a significant contribution of the APS, and for points $<1 \mathrm{~km}$ apart, a variance $<0.1 \mathrm{rad}^{2}$ is common. ${ }^{16,17}$ Therefore, the contribution of the APS to the double-difference phase for arcs of a shorter length than a threshold value can be neglected, and the value of $\varphi_{\mathrm{atmo}, i j}^{k}$ is assumed to be zero. Moreover, the influence of the noise on the time series of each arc is minimized by applying a low-pass filtering method. The principal goal is thus to estimate the relative constant velocity and integer ambiguity from the wrapped phase for all arcs in all interferograms, assuming a constant-velocity model for target motion.

To estimate the unknown parameters, i.e., the relative deformation rate and phase ambiguities, we use the temporal unwrapping method presented in DePSI. ${ }^{1,2}$ Because the ambiguity parameter is an integer, the approaches that can be used for integer ambiguity estimation and temporal unwrapping are the ambiguity function, ${ }^{10}$ integer bootstrapping,${ }^{18}$ integer least square, ${ }^{18}$ and LAMBDA ${ }^{18}$ methods. In this study, we used the LAMBDA method for temporal unwrapping that maximizes the probability of the correct estimation of the integers (success rate) and can yield the optimal method for the estimation of the deformation rates. This approach can be applied quickly, and it is capable of using any variance-covariance matrix of observations. ${ }^{18}$ Equation (2) for $N$ interferograms is rewritten as follows by ignoring the terms of the APS, nonlinear, and noise components:

$$
E\left\{y=\left[\begin{array}{c}
\psi_{i j}^{1} \\
\vdots \\
\psi_{i j}^{N} \\
V_{i j}
\end{array}\right]\right\}=\left[\begin{array}{lll}
-2 \pi & & \\
& \ddots & \\
& & -2 \pi
\end{array}\right]\left[\begin{array}{c}
a_{i j}^{1} \\
\vdots \\
a_{i j}^{N}
\end{array}\right]+\left[\begin{array}{c}
\frac{-4 \pi}{\lambda} T^{1} \\
\vdots \\
\frac{-4 \pi}{\lambda} T^{N} \\
1
\end{array}\right]\left[V_{i j}\right]
$$

and

$$
D\{y\}=Q_{y} .
$$

Equation (3) shows the functional model in which $E\{\cdot\}$ is the expectation operator. The system is nonlinear because the unwrapped phases are not available. Insofar as each doubledifference wrapped phase has its own unknown ambiguity, in addition to its unknown velocity, pseudo-observations for the displacement parameter must be added to solve for the rank deficiency of the system of equations. ${ }^{2,18}$ The stochastic model is illustrated in Eq. (4), where $D\{\cdot\}$ is the dispersion. The variance-covariance matrix, $Q_{y}$, is composed of the variance-covariance matrix of the phase observations estimated using the variance component estimation (VCE) according to Ref. 1 and the variance of the pseudo-observations achieved from the a priori standard deviation of the unknown parameters.

The LAMBDA method is based on the integer least-squares approach using a minimization problem. The key element of the LAMBDA method is based on reparameterizing the integer least-squares problem such that the equivalent problem is obtained while the ambiguities decorrelate. If the ambiguities were transformed and completely decorrelated in the first step, the problem would be much easier to solve. The computation of the best integer values for the transformed ambiguities according to the minimization process is the second step of the LAMBDA method. ${ }^{18}$ After parameter estimation using the LAMBDA method, the ambiguities and constant velocities that are relative in space are spatially unwrapped to obtain the absolute values, however, with regard to a reference PS. If the residual phases (the differences between the observations and the modeled double-difference phases) resulting from the temporal unwrapping were zero, we could assume that the ambiguities were correctly estimated. However, non-zero residuals are found at the arcs of the network due to noise, model imperfections, and incorrect relative estimations at certain arcs, which are considered as unwrapping errors. ${ }^{14,15}$ Therefore, the unwrapping error should be detected. As discussed in Refs. 2 and 14, the arcs with very low temporal coherence estimates showing large least-squares residuals are first eliminated. In the next step, the PS pixels that are connected by fewer than three arcs are removed. Consequently, 
some of the selected PS pixels are removed, hereafter called removed-PS. Therefore, an estimate for the ambiguities of the remaining PS pixels, called remnant-PS, is obtained from the ambiguities of the remaining arcs. In the final step, the constant velocities of the remnant-PS pixels are estimated using the obtained unwrapped phases.

\subsection{Approximation of the APS and the Nonlinear Component of the Deformation}

If the spatially uncorrelated portion of the look angle error and the linear component of deformation in steps 4 and 5 of Fig. 2 are estimated correctly, the residual phase as the result of the previous step will include atmospheric delay, orbital errors, nonlinear deformation, and noise. The residual phases of all of the remnant-PS pixels per interferogram can be separated into the APS, the nonlinear component of deformation, and random noise, assuming that the atmospheric signal is uncorrelated in time but correlated in space, whereas the unmodeled deformation is correlated in both time and space. The noise is uncorrelated in both time and space. The APS of the master, which is equal to the average of the residual phases in time, for each remnantPS is first subtracted from the initial residual phase. A temporal high-pass filter is then applied to the residual phase of all of the remnant-PS pixels to separate the APS and noise from the nonlinear deformation. ${ }^{19}$ Finally, Kriging interpolation, acting as a noise removal filter, is employed to spatially interpolate the phase of the APS and the nonlinear deformation for all of the removed-PS.

\subsection{Final Estimate for the Linear Component of the Deformation}

After the approximation and subtraction of the phases due to the APS and the nonlinear deformation for each PS per interferogram from the observed phase, the linear component of the deformation should be re-estimated (step 7). Therefore, step 5 needs to be repeated at this stage in which the observations are modified. This step improves the final estimation accuracy.

\section{Experimental Results}

The EPSI method was applied to estimate the deformation rate in the southwestern Tehran basin undergoing land subsidence due to the overexploitation of groundwater. The Tehran basin is located in the north of Iran, between the Alborz Mountains to the north and the Arad and Fashapouye mountains to the south. The available data consisted of 22 descending ENVISAT ASAR images of track 149, acquired between July 18, 2003, and October 24, 2008 (see Fig. 3). Because the EPSI method is time consuming, three different subsets of the study area, with extents of $4.5 \times 2.7 \mathrm{~km}, 5 \times 3.4 \mathrm{~km}$, and $4.5 \times 3.6 \mathrm{~km}$ (rectangles a, b, and c, respectively, in Fig. 1), were selected for the implementation. The first subset was subject to the highest deformation rate in the Tehran basin. Therefore, it was the most suitable subset with which to assess the efficiency of the EPSI algorithm; hereafter, this subset is called the first study

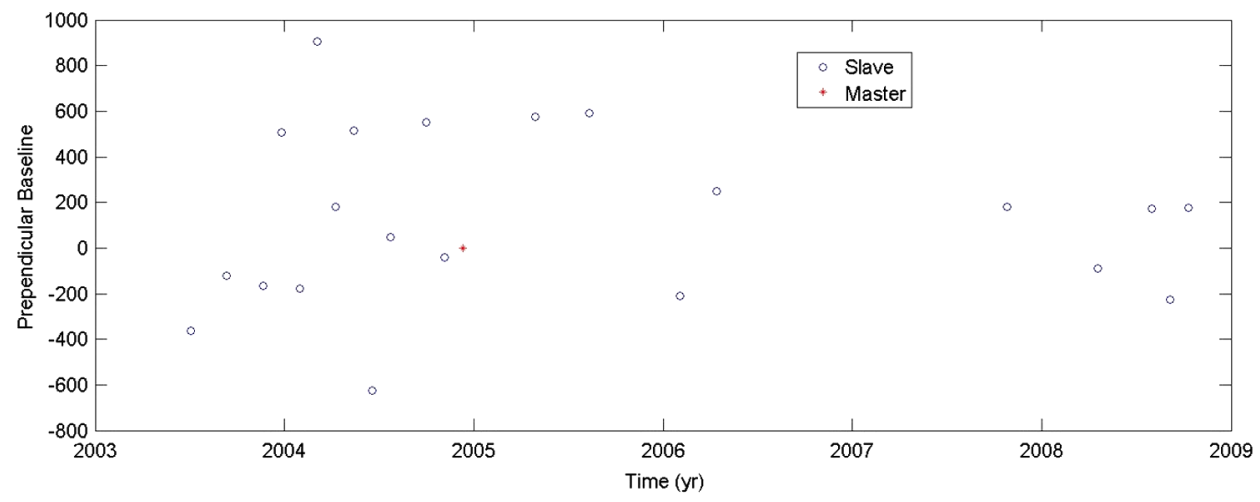

Fig. 3 Acquisition geometry of the available data in the area: temporal baselines versus spatial baselines. 
area, and the other subsets (rectangles b and c, respectively, in Fig. 1) are called the second and third. The master image was selected to maximize the stack coherence, which is a function of the temporal and perpendicular baselines and the Doppler centroid frequency. Twenty-one interferograms were then processed using the image acquired on December 24, 2004, as the master image. The Shuttle Radar Topography Mission (SRTM) digital elevation model (DEM) was used to remove the primary topographic contribution to the interferometric phase.

StaMPS was applied to select the PS pixels based on the amplitude dispersion with a threshold of 0.4 and phase analysis. As a result, 680, 624, and 296 PS pixels were identified in the first, second, and third study areas, respectively. The distribution of the PS pixels shown in Fig. 4 is not homogeneous because some regions lack PS pixels. After the estimation and subtraction of $\Delta \phi_{\theta, x, i}^{u}$ and $\phi_{x}^{m, u}$, the PS pixels were connected to form a network using Delaunay triangulation in which the maximum distance was limited to $2 \mathrm{~km}$ to reduce the atmospheric effect.

The standard deviation of the displacement rate per arc was set at $0.05 \mathrm{~m} /$ year in the first study area and $0.03 \mathrm{~m} /$ year in the second and third study areas to construct the variance of the pseudo-observation and estimate the variance-covariance matrix of the phase observations using VCE. Note that this value has a significant influence on the estimated integer ambiguities and the deformation rates. After the estimation of the relative velocity and integer ambiguities per arc, an a posteriori variance factor was estimated as $\hat{\sigma}=\hat{n} Q_{y}^{-1} \hat{n} / r$ (where $\hat{n}$ and $r$ are the vector of leastsquares residuals per arc for all interferograms and the redundancy, respectively). ${ }^{18}$ Large deviation between the observations and the predefined deformation model, as in regions suffering from a high nonlinear deformation mechanism, causes error in the estimated ambiguities, resulting in large values of $\hat{n}$ and $\hat{\sigma}$. As it is formulated, $\hat{\sigma}$ is dependent on $Q_{y}$, and a $\hat{\sigma}$ value of 1.0 indicates that the covariance matrix used in the estimation process correctly describes the dispersion of the observations, ${ }^{14}$ which depends on the selected value of the standard deviation of the displacement rate. Therefore, we removed arcs with $\hat{\sigma}>$ threshold. The threshold value here was set at 3.2.

After removing arcs with a high a posteriori variance factor, the number of remnant-PS and removed-PS pixels became 573 and 107, respectively, in the first study area, 520 and 104 in the second study area, and 245 and 51 in the third study area. The integer ambiguities and the deformation velocities were then obtained for all of the remnant-PS pixels in each study area. After the APS and nonlinear deformation estimation and removal of all of the remnant-PS pixels, these contributions were predicted for the removed-PS pixels. The steps were repeated once, beginning with forming a network up to approximating the APS and the nonlinear deformation, with the phases compensated for the APS and the nonlinear deformation.

Figure 5 shows the velocity map of the study areas from the EPSI algorithm. The maximum rates estimated for the three different subsets of the Tehran basin were 24.95, 15, and $14.5 \mathrm{~cm} /$ year. The deformation time series of the selected PS pixels (as illustrated in Fig. 5), located in different parts of the study areas, are illustrated in Fig. 6. The deformation time series of these points show that the subsidence behavior is linear without significant seasonal fluctuations.

\section{Validation of the Results}

To quantitatively evaluate the results in the absence of the ground-truth data, i.e., the leveling measurements taken exactly within the temporal interval of the radar data used in this study (2003-2008), the available leveling measurements collected in the study areas in February 2005 and February 2006 by the National Cartographic Center (NCC) were used. ${ }^{20,21}$ In the first, second, and third study areas, there are 6,8, and 5 leveling stations, respectively, with second- and third-order accuracy as observed in Fig. 5. The comparison between the results obtained from the proposed method and the leveling measurements is summarized in Fig. 7. The leveling measurements are projected along the line of sight direction, assuming an incidence angle of $23 \mathrm{deg}$. As the figures show, the spatial pattern of the subsidence extracted from the proposed algorithm is highly similar to the leveling measurements along the leveling stations. However, the different deformation rate of the two datasets was most likely due to the different time intervals covered by the radar data (from 2003 to 2008) and by the leveling measurements (2005). 


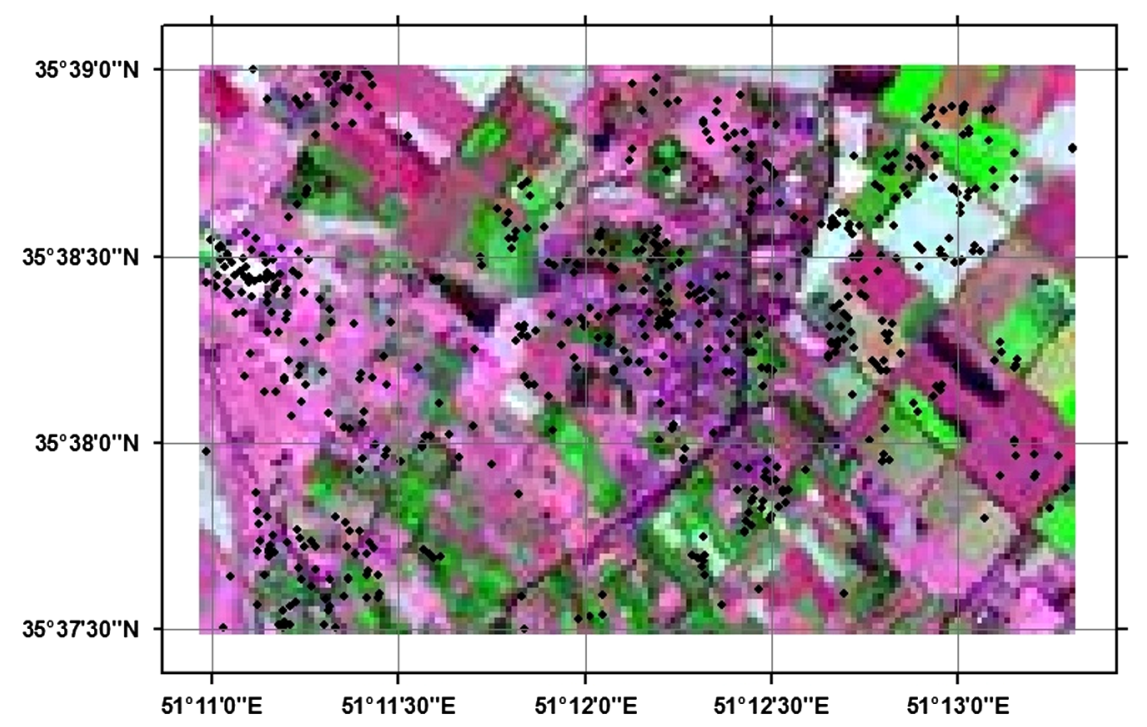

(a)

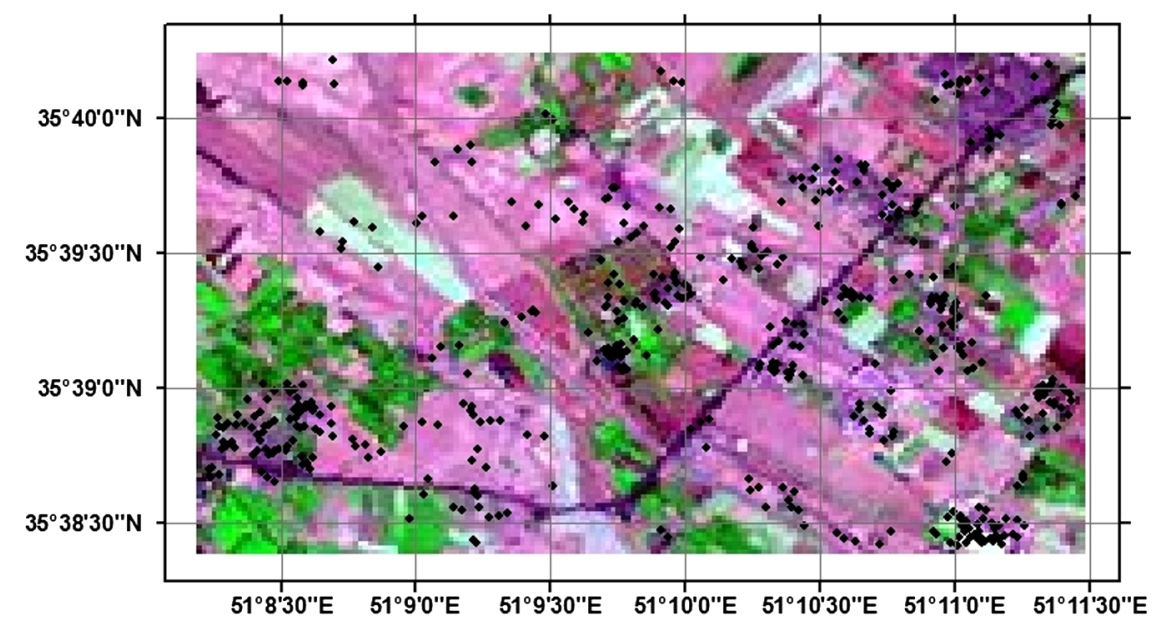

(b)

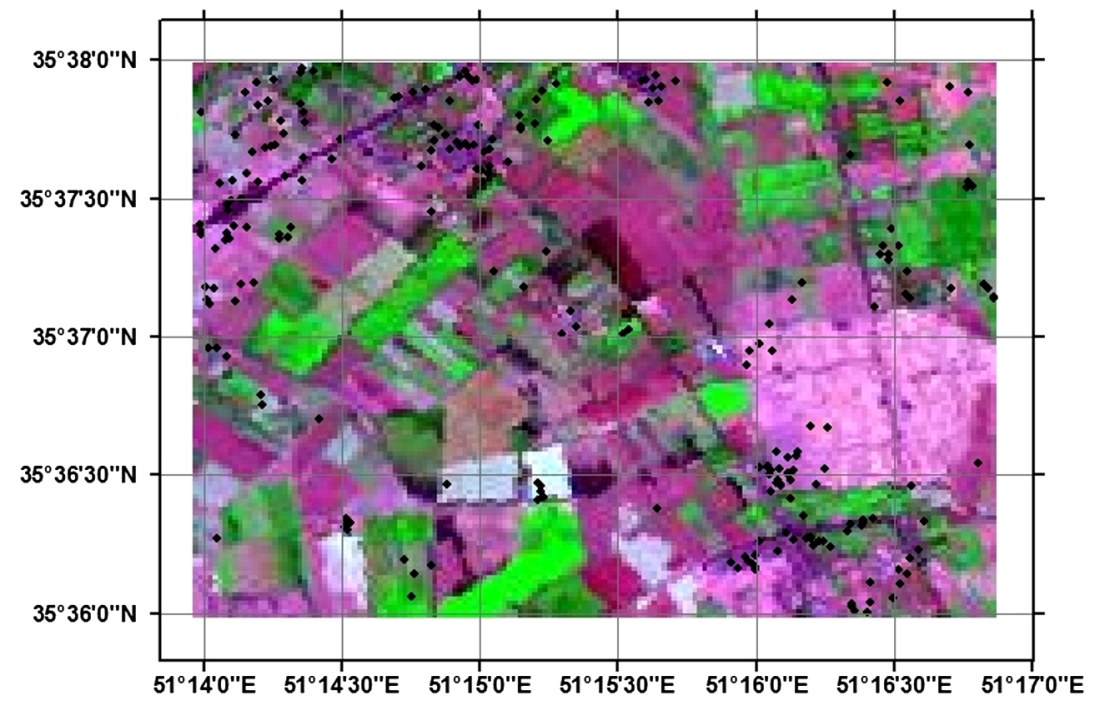

(c)

Fig. 4 Geographical location of the first study area (a), the second study area (b), and the third study area $(\mathrm{c})$ in the Tehran basin based on the LANDSAT $7 \mathrm{ETM}^{+}$color composite image (R:7, G:4, B:2). The black circles show the position of the selected persistent scatterer (PS) pixels in the study area. 


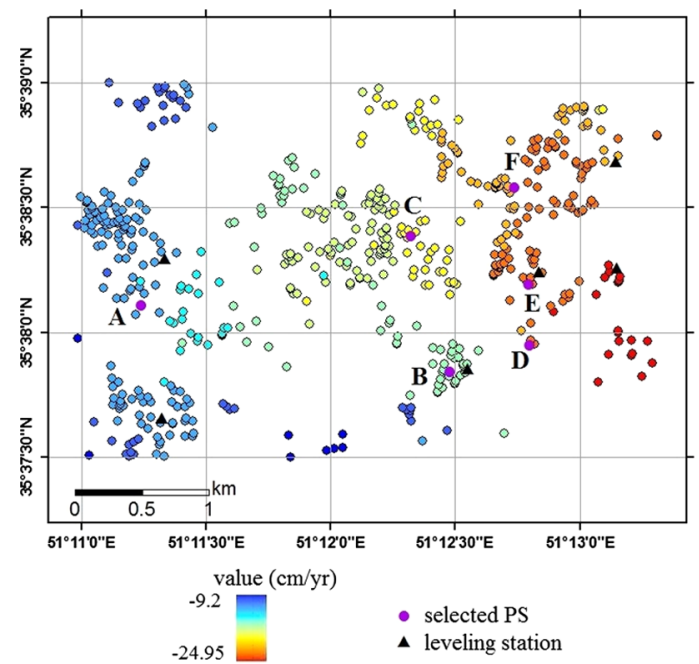

(a)

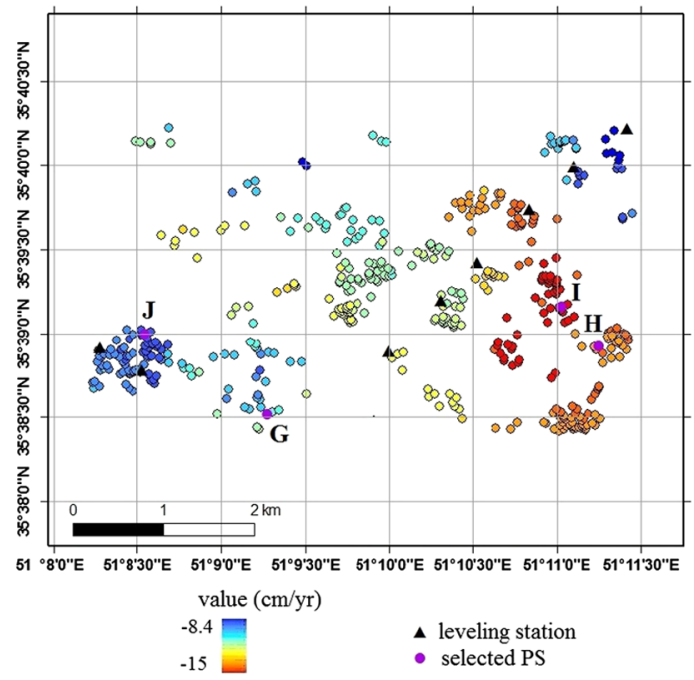

(b)

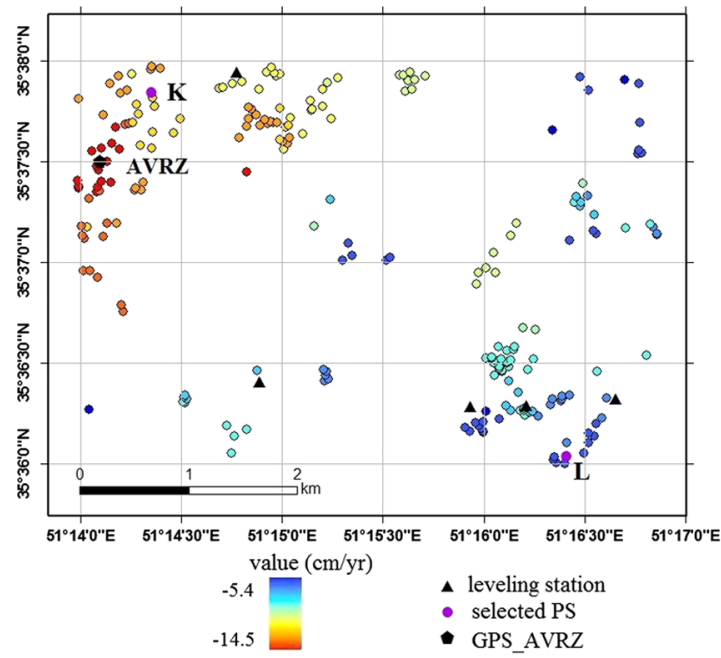

(c)

Fig. 5 Mean velocity map of the first study area (a), the second study area (b), and the third study area (c). Purple circles A-L represent the locations of the PS pixels for which the deformation time series is shown in Fig. 6 . The black triangles illustrate the leveling stations. 

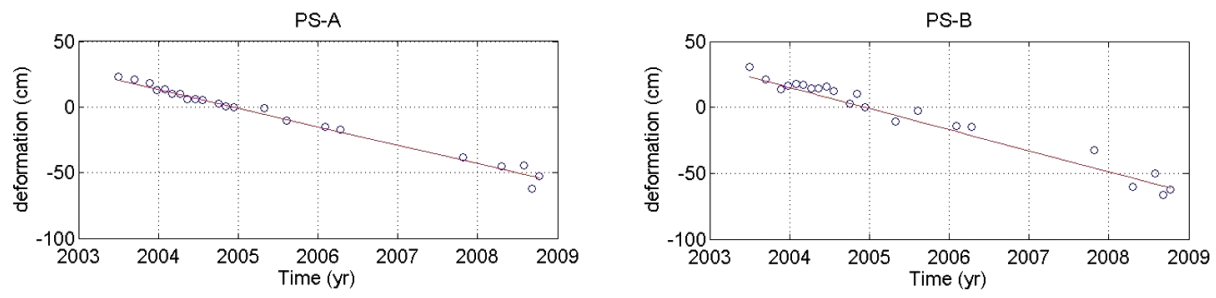

- time serie

estimated velocity
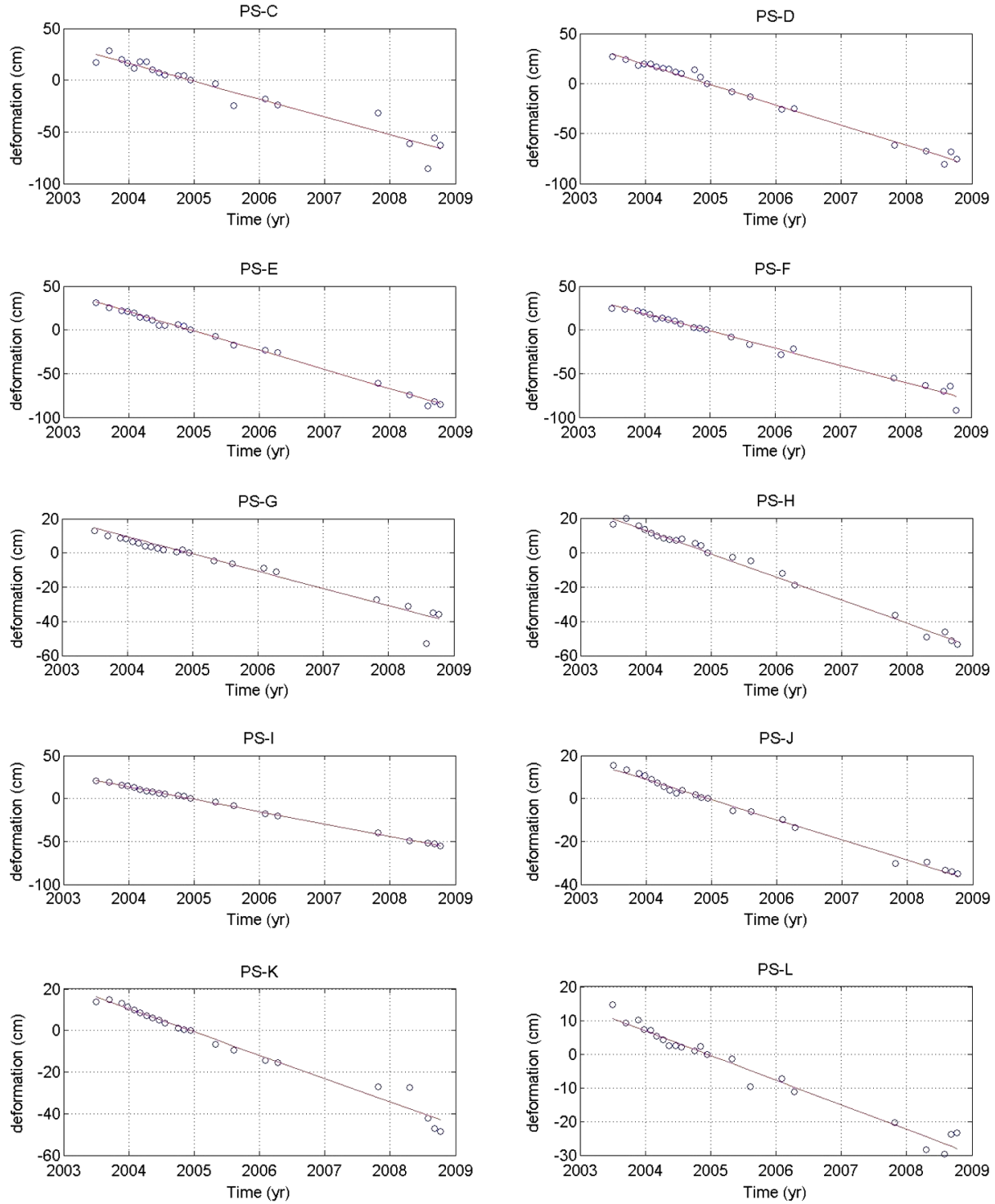

Fig. 6 The deformation time series of the selected PS in Fig. 5 (A-L) with regard to the October 24, 2004, master image [after the atmospheric phase screen (APS) and nonlinear deformation removal].

In the first study area, the root mean square error (RMSE) between the leveling measurements and the EPSI results was $2 \mathrm{~cm} /$ year. Across the leveling stations, the maximum deformation rate estimated from the standard StaMPS was $2.5 \mathrm{~cm} /$ year, whereas the true maximum subsidence rate was approximately $20 \mathrm{~cm}$ /year according to the leveling measurements. The RMSE between the leveling measurements and the results obtained from the standard StaMPS was $14.9 \mathrm{~cm} /$ year. 
Sadeghi et al.: Enhanced algorithm based on persistent scatterer interferometry...

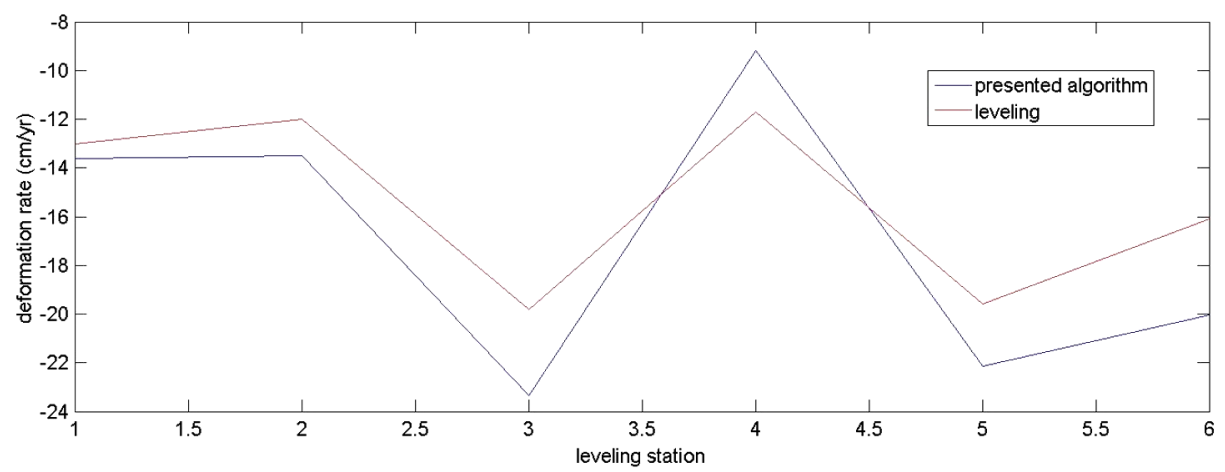

(a)

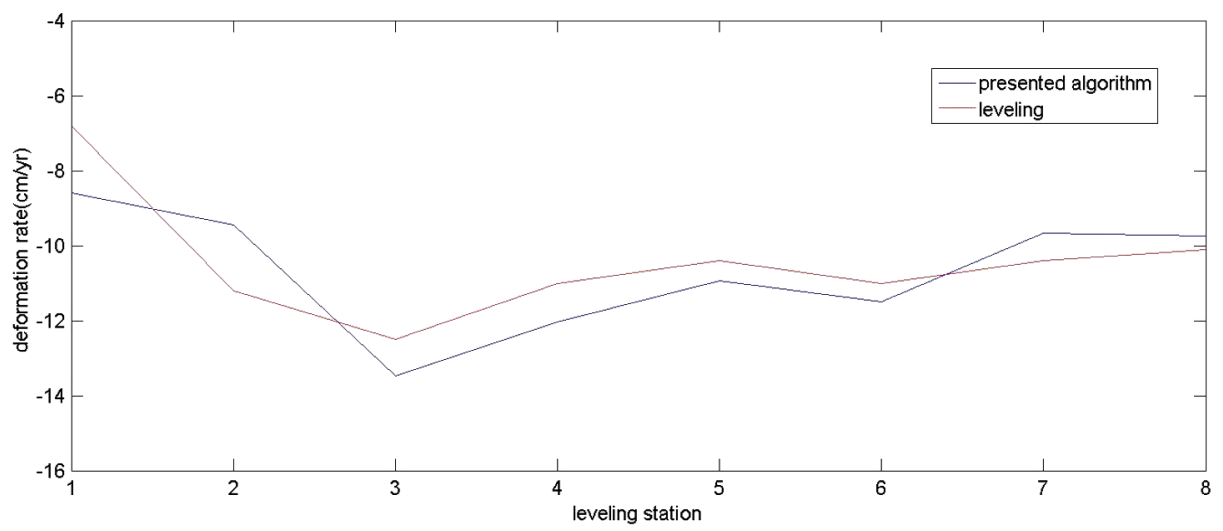

(b)

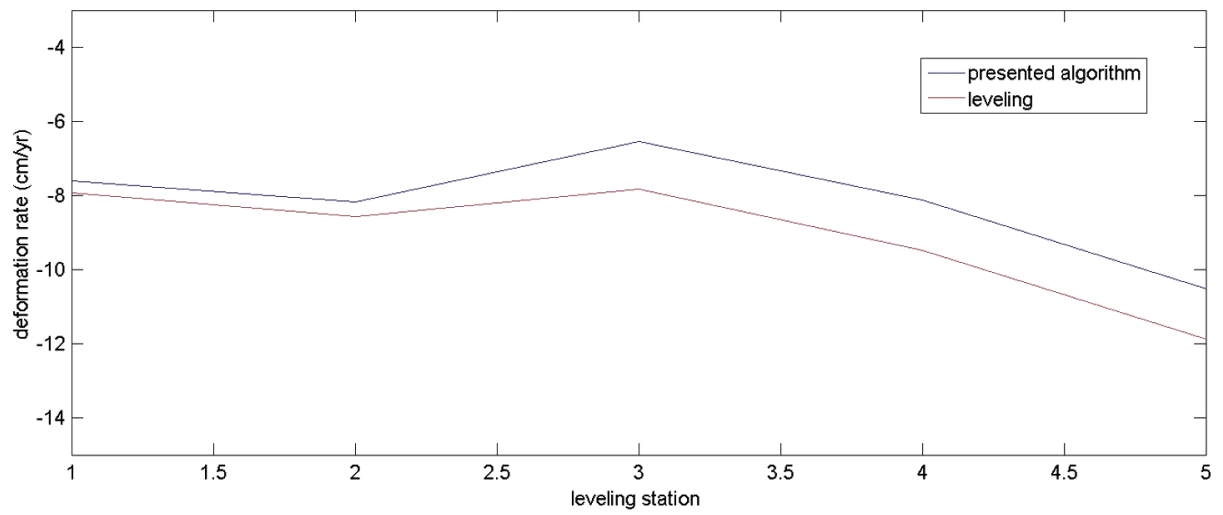

(c)

Fig. 7 Comparison of the subsidence rates inferred from enhance persistent scatterer interferometry (EPSI) and leveling in the first (a), second (b), and third (c) study area along the line-of-sight direction.

In the second and third study areas, the RMSE between the leveling measurements and the EPSI results was $1.1 \mathrm{~cm} /$ year, whereas the RMSEs between the leveling measurements and the results of the standard StaMPS were 9.5 and $8.4 \mathrm{~cm} /$ year, respectively. The comparison between the RMSEs obtained from the EPSI method and those resulting from the standard StaMPS algorithm demonstrated that the proposed method has significantly improved the performance of PSI. This fact confirmed the ability of the EPSI when compared with other interferometry methods to extract the deformation rate for a difficult test case, i.e., a rural area with a high deformation rate and poor temporal sampling of the available radar data.

However, if the time coverage between the leveling measurements and the radar data were the same, we would be able to more precisely evaluate the results. In addition to a different time interval, another reason for the difference between the results obtained from the EPSI and leveling measurements is probably the local unwrapping errors. There are several limiting factors that 


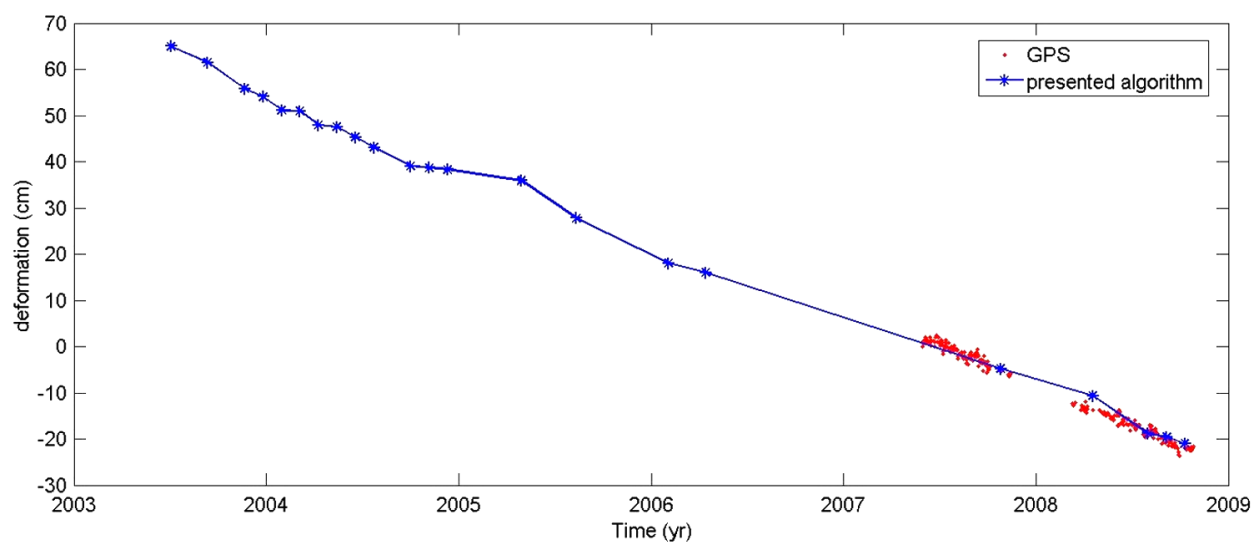

Fig. 8 Comparison of the time series of the GPS and the algorithm presented (InSAR) at the AVRZ station.

decrease the phase-unwrapping performance of the enhanced algorithm: (1) Partially poor temporal sampling of the radar data causes the unwrapping error, biasing the estimates of the deformation rate toward the values obtained from a portion of the data with better temporal sampling. Moreover, due to the poor sampling of the data in time, the nonlinear component of the deformation cannot be reliably separated from the atmospheric signal of the slave images when applying a high-pass filter in time. (2) An inappropriate value of the standard deviation of the pseudoobservations affects the parameter estimations. (3) The low density of the PS pixels causes the incorrect estimation of the APS contribution to the double-difference phase, resulting in an unwrapping error.

To evaluate the results of the time-series analysis, continuous GPS measurements were used. One GPS station (AVRZ) was established by NCC as a part of the Iranian Permanent Network for Geodynamics (IPGN) program in the third study area, as illustrated in Fig. 5 . $^{22}$ This station recorded data continuously from June 13, 2007, to November 8, 2008, with a gap between 2007 and 2008. The InSAR time series data collected at the location of the AVRZ station, in addition to the GPS measurements, are shown in Fig. 8. The InSAR results demonstrated a good agreement with the GPS measurements for the period of their overlap. The RMSE between the GPS measurements and the InSAR time series results was estimated to be $1.11 \mathrm{~cm}$.

Although the efficiency of the enhanced algorithm is affected by the above factors, the results show that the enhanced algorithm in this study is able to measure the displacement in a rural area with a high deformation rate, which is a significant improvement in the PSI approach.

\section{Conclusions}

In this paper, we introduced an enhanced algorithm that integrates the advantages of two different PSI methods to estimate the high deformation rate in a rural area lacking bright scatterers. One of the PSI methods, DePSI, cannot detect the PS pixels in a rural area lacking high-amplitude human-made structures. In contrast, the second PSI method, StaMPS, can successfully identify PS pixels in rural areas without assuming an a priori deformation model; however, it underestimates the deformation rate when it is too high relative to the sampling of the data in time because it uses the Nyquist sampling assumption in the phase-unwrapping step. In the proposed algorithm, the PS pixels are first selected by the amplitude analysis and phase stability estimation as used in StaMPS. The deformation rate and ambiguity parameter are then estimated using the LAMBDA method employed in DePSI.

The enhanced algorithm was applied to the descending dataset of the Tehran basin, given that none of the current PSI techniques can be correctly applied to the area. The available data consisted of 22 ENVISAT ASAR images of track 149 from 2003 to 2008. The maximum deformation rates estimated in three different subsets of the study area were $24.95,15$, and $14.5 \mathrm{~cm} /$ year. The deformation time series at the different pixels indicate that the ground surface is continuously subsiding at a constant rate without significant seasonal fluctuations. 
The results obtained from the enhanced algorithm were compared with the leveling and GPS measurements. The different deformation rates extracted from the proposed method and the leveling and GPS measurements likely resulted from the different time intervals covered by the radar data and the leveling and GPS measurements. However, the RMSE between the InSAR and the leveling and the GPS measurements demonstrates that the results of the enhanced algorithm are significantly improved compared with those based on the standard StaMPS. Ultimately, this method in this study is able to measure the high deformation rate in rural areas even if the only available radar data have poor temporal sampling, clearly indicating a significant improvement in the implementation of PSI. Yet more advanced analyses related to the different error sources and limiting factors may be considered for future work.

\section{Acknowledgments}

The authors are thankful for the European Space Agency for providing the ENIVSAT ASAR data. We also wish to express our sincere gratitude to the National Cartographic Center of Iran for collecting the time series Global Positioning System measurements and precise leveling data.

\section{References}

1. B. M. Kampes, "Displacement parameter estimation using permanent scatterer interferometry," Ph.D. thesis, (Delft University of Technology, Delft, The Netherlands 2005).

2. V. B. H. Ketelaar, "Monitoring surface deformation induced by hydrocarbon production using satellite radar interferometry," Ph.D. thesis, (Delft University of Technology, Delft, The Netherlands 2008).

3. A. Hooper, "Persistent scatterer RADAR interferometry for crustal deformation studies and modeling of volcanic deformation," Ph.D. thesis, (Stanford University, Stanford, CA 2006).

4. A. Hooper, P. Segall, and H. Zebker, "Persistent scatterer interferometric synthetic aperture radar for crustal deformation analysis with application to Vacan Alcedo, Galapagos," J. Geophys. Res. 112, B07407 (2007a), http://dx.doi.org/10.1029/2006JB004763.

5. A. Hooper and H. Zebker, "Phase unwrapping in three dimensions with application to InSAR time series," J. Opt. Soc. Am. A 24 (9), 2737-2747 (2007b), http://dx.doi.org/10 .1364/JOSAA.24.002737.

6. M. Dehghani et al., "Hybrid conventional and persistent scatterer SAR interferometry for land subsidence monitoring in Tehran basin, Iran," Fringe 09 in Proc. of Workshop on Advances in Science and Applications in SAR interferometry, ESA ESRIN, Frascati, Italy (2009).

7. M. Dehghani, "Estimation of deformation rate and modeling of land subsidence induced by groundwater exploitation using interferometry," Ph.D. thesis, (K. N. Toosi University, Tehran, Iran 2010).

8. F. Rocca, "Modeling interferogram stacks," IEEE Trans. Geosci. Rem. Sens. 45 (10), 3289-3299 (2007), http://dx.doi.org/10.1109/TGRS.2007.902286.

9. U. Wegmuller et al., "Nonuniform ground motion monitoring with TerraSAR-X persistent scatterer interferometry," IEEE Trans. Geosci. Rem. Sens. 48 (2), 895-904 (2010), http://dx .doi.org/10.1109/TGRS.2009.2030792.

10. A. Ferretti, C. Prati, and F. Rocca, "Permanent scatterers in SAR interferometry," IEEE Trans. Geosci. Rem. Sens. 39 (1), 8-20 (2001), http://dx.doi.org/10.1109/36.898661.

11. M. Dehghani et al., "Interferometric measurements of ground surface subsidence induced by overexploitation of groundwater," J. Appl. Remote Sens. 4 (1), 041864 (2010), http://dx .doi.org/10.1117/1.3527999.

12. M. Dehghani et al., "InSAR monitoring of progressive land subsidence in Neyshabour; Northeast Iran," Geophys. J. Int. GJI 178, 47-56 (2009a), http://dx.doi.org/10.1111/gji 2009.178.issue-1.

13. M. Dehghani et al., "RADAR interferometry time series analysis of Mashhad subsidence," J. Int. Soc. Rem. Sens. 37(1), 147-156 (2009b), http://dx.doi.org/10.1007/s12524-009 $-0006-\mathrm{x}$. 
14. S. Samiei Esfahany, "Improving persistent scatterer interferometry results for deformation monitoring," M.S. thesis, (Delft University of Technology, Delft, The Netherlands 2008).

15. A. J. M. Humme, "Point density optimization for SAR interferometry: a study tested on salt mine areas," M.S. thesis, Department of Earth Observation and Space Systems, Faculty of Aerospace Engineering, (Delft University of Technology, Delft, The Netherlands 2007).

16. S. Williams, Y. Bock, and P. Fang, "Integrated satellite interferometry: tropospheric noise, GPS estimates and implications for interferometric synthetic aperture radar products," J. Geophys. Res. 103(B11), 27,051-27,067 (1998), http://dx.doi.org/10.1029/98JB02794.

17. C. Colesanti et al., "Monitoring landslides and tectonic motions with the permanent scatterers technique," Eng. Geol. 68, 3-14 (2003), http://dx.doi.org/10.1016/S0013-7952(02) 00195-3.

18. B. M. Kampes and R. F. Hanssen, "Ambiguity resolution for permanent scatterer interferometry," IEEE Trans. Geosci. Rem. Sens. 42 (11), 2446-2453 (2004), http://dx.doi.org/10 $.1109 /$ TGRS.2004.835222.

19. A. Ferretti, C. Prati, and F. Rocca, "Nonlinear subsidence rate estimation using permanent scatterers in differential SAR interferometry," IEEE Trans. Geosci. Rem. Sens. 38 (5), 2202-2212 (2000), http://dx.doi.org/10.1109/36.868878.

20. M. Amighpey et al., "Elevation changes of the precise leveling tracks in the Iran leveling network," Scientific report published in National Cartographic Center (NCC) of Iran, Tehran (2006).

21. S. Arabi et al., "Study of subsidence of southwestern of Tehran basin," J. Nat. Cartogr. Cent. (NCC) Iran 16 (69), 14-28 (2005).

22. A. Soltanpoor et al., "Study of changes in southwestern Tehran basin from viewpoint of physical geodesy," Scientific report published in National Cartographic Center (NCC) of Iran, Tehran (2010).

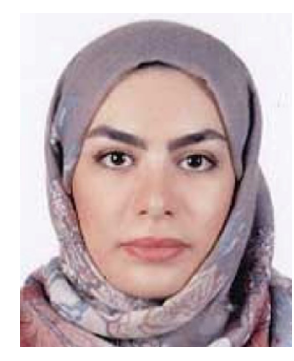

Zahra Sadeghi received her BS degree in geodesy and geomatics engineering from Shahid Rajaee University and MSc degree in remote sensing from K. N. Toosi University of Technology in 2008 and 2011, respectively in Tehran, Iran. Her main research interests are SAR interferometry, polarimetric SAR interferometry, and SAR tomography.

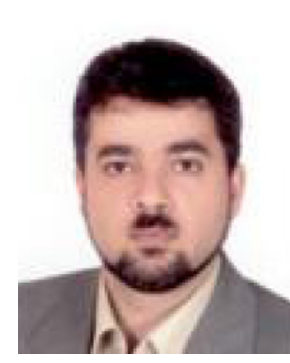

Mohammad Javad Valadan Zoej received his $\mathrm{PhD}$ degree in photogrammetry and remote sensing from the University of Glasgow, Glasgow, UK. $\mathrm{He}$ is currently an associate professor with the Faculty of Geodesy and Geomatics Engineering, K. N. Toosi University of Technology, Tehran, Iran. His research interests include 3D spatial information extraction from space images, automatic feature extraction from aerial and space images, and SAR interferometry.

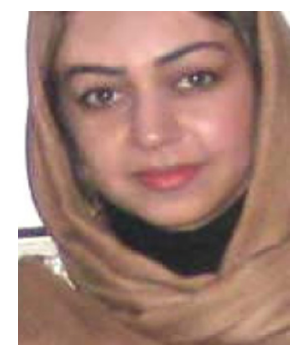

Maryam Dehghani received her BS degree in geodesy and geomatics engineering from Iran University of Science and Technology, and her MSc and $\mathrm{PhD}$ degrees in remote sensing from K. N. Toosi University of Technology in 2004 and 2009, respectively. During her PhD studies, she received scholarships to carry out research at the University of Oxford and the University of Delft. From 2002 to 2008, she was working in the Remote Sensing group of the Geological Survey of Iran (GSI). She is currently an assistant professor with the Department of Civil and Environmental Engineering, School of Engineering, Shiraz University. Her main research interests are synthetic aperture radar interferometry (InSAR) and digital image processing. 


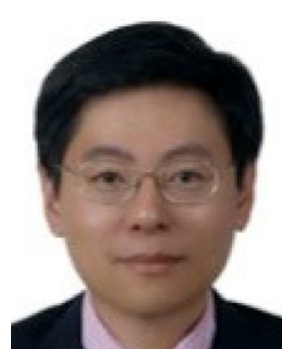

Ni-Bin Chang is currently a professor with civil and environmental engineering department and the director of the Stormwater Management Academy, University of Central Florida. He received his MS and $\mathrm{PhD}$ degrees in environmental systems engineering from Cornell University in 1989 and 1991, respectively. His areas of research include environmental and hydrological informatics, environmental cyberinfrastructure and remote sensing, and computational intelligence. He has been working with various funding agencies to carry out optical and microwave remote sensing through airborne and space-borne sensors for environmental monitoring and management. He is a senior member of the Institute of Electrical and Electronics Engineers (IEEE) and SPIE, a fellow of American Society of Civil Engineers (F.ASCE) and the American Association for the Advancement of Science (F.AAAS), and a member of the European Academy of Sciences (M.EAS). 